\title{
Latest developments in the field of stem cell research and regenerative medicine compiled from publicly available information and press releases from nonacademic institutions 1-30 June 2016
}

First draft submitted: 21 July 2016; Accepted for publication: 28 July 2016; Published online: 1 September 2016

\section{Business development}

\begin{abstract}
Collaborations, partnerships
\& alliances

\section{Cobranding agreement: Biological Industries \& Corning}

Biological Industries (BI; CT, USA; www.bioind.com) announced a cobranding agreement with Corning's subsidiary, Mediatech (NY, USA; www.corning.com and www.cellgro. com), which will enable cell therapy, research and manufacturing organizations around the world to purchase the xeno-free NutriStem ${ }^{\circledR}$ human pluripotent stem cell medium in conjunction with Corning's existing portfolio of stem cell-focused technologies. Before the end of this year, Corning and BI will launch a jointly branded NutriStem hPSC XF Medium, which will continue to be manufactured by BI, but marketed, distributed and supported worldwide by Corning's global commercial team.
\end{abstract}

\section{Codevelopment agreement: Cellular Dynamics International \& National Eye Institute}

Cellular Dynamics International (WI, USA; www.cellulardynamics.com), a FUJIFILM company (Japan; www.fujifilmholdings.com) has entered into a cooperative research and development agreement with the National Eye Institute (www.nei.nih.gov), a division of the NIH, to conduct research to advance a cell transplantation therapy for the treatment of retinal degenerative disease using retinal pigment epithelium derived from induced pluripotent stem cells (iPSCs). The collabo- ration includes the joint development of an optimized biocompatible biodegradable scaffold that is likely to be a critical component for a potential future therapy.

\section{Collaboration agreement: Orig3n \\ \& Kangstem}

ORIG3N (MA, USA; www.orig3n.com) and Kangstem Biotech (Korea; www.kangstem.com) announced the beginning of a scientific collaboration to study the utility of induced neural stem cells in disease modeling. As part of the collaboration, Kangstem Biotech will provide patient-specific induced neural stem cells differentiated directly from skin fibroblasts for ORIG3N to use in its disease-modeling platform.

\section{Licensing agreement: Bone Biologics \& University of California Los Angeles} Bone Biologics (MA, USA; http://bonebiologics.com) signed an option agreement with the University of California Los Angeles for an opportunity to exclusively license the use of the bone growth factor Nell-1 in the treatment of osteoporosis. Currently, Bone Biologics is funding the development and formulation of an Nell-1-based product for use as a bone graft substitute on bone regeneration in spinal fusion procedures. The new technology would consist of NELL-1, Bone Biologics' proprietary skeletal specific growth factor, used in combination with $\mathrm{DBX}^{\circledR}$, a proprietary demineralized bone matrix from Musculoskeletal Transplant Foundation (www.mtf.org/index.html).
Dusko Ilic*,1 \& Cristina Trento ${ }^{2}$ 'Stem Cell Laboratories, Guy's Assisted Conception Unit, Division of Women's Health, King's College London, London, UK

${ }^{2}$ Department of Haemato-Oncology, Rayne Institute, Faculty of Science \& Medicine, King's College London, London, UK

*Author for correspondence: dusko.ilic@kcl.ac.uk
Future $\%$
Medicine $\%$ part of fsg 
Licensing agreement: Emmaus \& Cell Seed

A licensing agreement between Emmaus Medical (CA, USA; www.emmausmedical.com) and CellSeed (Japan, www.cellseed.com/index-e.html) has been signed to commercialize Epithelial Cell Sheet Corneal Regeneration technology for limbal stem cell deficiency (LSCD) in USA. LSCD has been recognized as a major cause, either primary or secondary, of significant visual loss and blindness in many common corneal disorders. The new agreement extends Emmaus' existing relationship with CellSeed, allowing the company to continue to conduct research and development activities on the epithelial cell sheet for the treatment of LSCD. Emmaus will pay a royalty to CellSeed upon commercialization.

\section{Licensing agreement: TxCell \& Lübeck Institute of Experimental Dermatology}

TxCell SA (France, www.txcell.com/index.php/en/) signed a strategic R\&D collaboration agreement with the Lübeck Institute of Experimental Dermatology (Germany; www.lied.uni-luebeck.de/home.html). This specific collaboration agreement covers the development of a CAR-Treg-based cellular immunotherapy for bullous pemphigoid, a rare and potentially fatal autoimmune disease. TxCell scientists have already identified a relevant antigenic target for the development of a CAR-Treg product in bullous pemphigoid patients. The CAR construct will be designed to ensure the activation of CAR-Treg cells specifically in the inflammatory skin lesions. TxCell and Lübeck Institute of Experimental Dermatology will conduct nonclinical pharmacology studies with CAR-Treg cells to prepare for a first in-man study in bullous pemphigoid patients. TxCell retains all rights on existing and future programs and products developed under this agreement. Financial terms of the collaboration have not been disclosed.

\section{Licensing agreement: TxCell \& Yeda}

The European Patent Office has granted Yeda Research and Development (Israel; www.yedarnd.com) the patent covering all redirected, genetically engineered Tregs (CAR-Tregs) and their use in the suppression of autoimmune and inflammatory diseases (patent identification number: EP 2126054). TxCell has signed an exclusive worldwide licensing agreement with Yeda Research and Development, owner of the patent, to develop and commercialize CAR-Treg products for the treatment of autoimmune and inflammatory diseases, as covered by the patent family.

\section{Licensing \& collaboration agreement: CRISPR \&} Anagenesis

CRISPR Therapeutics (Switzerland; http://crisprtx. com) and Anagenesis Biotechnologies (France, http:// anagenesis-biotech.com) announced a strategic inlicensing and collaboration agreement to develop CRISPR/Cas9-based cell therapies for Duchenne muscular dystrophy. Anagenesis Biotechnologies' proprietary Paraxial Mesoderm Multipotent Cells technology, which allows differentiation of pluripotent cells, iPSC or embryonic stem cells (ESCs), into satellite cells, will support the advancement of CRISPR-based cellular therapies for the treatment of musculoskeletal diseases.

\section{Manufacturing agreement: Amarantus \& Lonza}

Amarantus BioScience Holdings (CA, USA; www. amarantus.com) announced that the current good manufacturing practices (cGMPs) manufacturing process for its engineered skin substitute (ESS) program is now qualified at Lonza Walkersville (MD, USA; www.lonza.com/custom-manufacturing/capabilitiesoverview/our-sites/walkersville-md-usa.aspx). ESS is a combination of cultured epithelium with a collagenfibroblast implant that produces a skin substitute that contains both epidermal and dermal components made from a patient's own skin and expanded to provide permanent wound coverage of large body surface areas. A non-GMP version ESS has already been used in investigator-initiated and compassionate-use clinical settings in over 150 patients, primarily pediatrics, for the treatment of severe burns up to $95 \%$ of total body surface area and also in the treatment of two patients with Giant Congenital Melanocytic Nevi. Amarantus can now commence its 10-patient, randomized, placebo-controlled, open-label Phase II clinical trial (clinicaltrials.gov identifier: NCT01655407) for the treatment of adult patients (ages 18-40 years old) with deep-partial and full-thickness thermal burns covering $\geq 50 \%$ of total body surface area. The study will compare the safety and efficacy of the autologous ESS with meshed, split-thickness autograft.

\section{Marketing agreement: StemBioSys \& Funakoshi} StemBioSys (TX, USA; www.stembiosys.com) has signed a 3-year marketing and distribution deal with Funakoshi (Japan; www.funakoshi.co.jp/exports) to bring its stem cell production products to Japan. StemBioSys develops an extracelluar matrix from bone marrow stem cells. The matrix is made of natural proteins that are created by stem cells to act like a home, helping the cells divide, expand and replicate.

\section{Partnering agreement: Cell Medica \& Baylor}

Cell Medica (UK; https://cellmedica.co.uk) and Baylor College of Medicine (TX, USA; www.bcm.edu) will partner to develop a pipeline of next-generation cellular immunotherapy products designed to fight cancer by attacking solid tumors. Baylor has provided 
Cell Medica with an exclusive license to its proprietary natural killer T-cell immunotherapy platform, five product candidates to be developed by Baylor research teams specializing in genetically engineered immune cells and an option to license future product candidates. The partnership includes a codevelopment component designed to combine Baylor's expertise in the creation of modified immune cell technologies with Cell Medica's know how in manufacturing and commercializing cell therapy products.

\section{Research agreement: Bellicum \& LUMC}

Bellicum Pharmaceuticals (TX, USA; www.bellicum. com) announced a research agreement with Leiden University Medical Center (LUMC; The Netherlands; www.lumc.nl) to discover and validate natural highaffinity T-cell receptor (TCR) product candidates targeting several cancers. The new collaboration builds on an earlier agreement with LUMC that gave Bellicum worldwide rights to TCR product candidates targeting solid tumors that express preferentially expressed antigen in melanoma and other antigens. Under terms of the new collaboration, Bellicum will provide financial support to LUMC over a 3-year term in exchange for the right to exclusively license any high-affinity TCRs discovered under the agreement. Bellicum's first TCR product candidate, BPX701 targeting preferentially expressed antigen in melanoma, is expected to enter Phase I/II clinical trials in mid-2016 to treat refractory acute myeloid leukemia and myelodysplastic syndromes. BPX701 was licensed from Leiden in 2015 and incorporates Bellicum's proprietary safety mechanism, CaspaCIDe, for improved control over the cells.

\section{Launching new projects, products \& services Allo}

AlloSource (CO, USA; www.allosource.org) launched two new innovative demineralized cortical fiber allografts, AlloFuse ${ }^{\circledR}$ Cortical Fibers and the AlloFuse Fiber Boat, for use in a variety of spine and orthopedic procedures. The demineralized bone fiber (DBFTM) technology used in these products is patented by TheraCell (MA, USA; www.theracellinc.com/index. php), partner of AlloSource. These new demineralized bone fiber allografts consist of $100 \%$ demineralized cortical bone and offer a less costly alternative to synthetic bone void fillers. The size and geometry of the fibers have been optimized to provide exceptional handling characteristics for bone fusion procedures, primarily in spine and orthopedics.

\section{DiscoveryBioMed}

DiscoveryBioMed (AL, USA; www.discoverybiomed. com) was awarded a new 3-year laboratory to Mar- ketplace Small Business Innovation Research grant of US\$1.2 million to fund the establishment of novel diseased and normal renal cell platforms. The award comes from the Office of the Director at the NIH. DiscoveryBioMed is seeking to establish single cyst-derived primary cultures from diseased kidney but also primary human cell cultures from normal kidneys in order to immortalize cultures for expansion for larger initiatives such as therapeutics discovery. This would benefit therapies for polycystic kidney disease and, in particular, for the more common autosomal dominant genetic form.

\section{Clinical trials}

\section{Cellectis}

Cellectis (France; www.cellectis.com/en/) announced that the first patient has been treated in the Phase I study of UCART19 in pediatric acute B-lymphoblastic leukemia at the University College of London (UK). This UCART19 clinical trial is sponsored by Servier (France; www.servier.com) in close collaboration with Pfizer (NY, USA; www.pfizer.com). The pediatric Phase I is an open-label, noncomparative, monocenter study to evaluate the safety and ability of UCART19 to induce molecular remission in pediatric patients with relapsed or refractory CD19-positive B-cell acute lymphoblastic leukemia ahead of planned allogeneic hematopoietic stem cell transplantation. Cellectis will receive a milestone payment from Servier of an undisclosed amount. The company has also recently been selected as a 2016 World Economic Forum Technology Pioneer, based on its contribution to and impact on the global healthcare and life sciences industries via the development and therapeutic application of allogeneic CAR T-cell immunotherapies targeting cancer.

\section{Celyad}

Celyad (Belgium, www.celyad.com) announced results for CHART-1, its European Phase III clinical trial for its lead cardiovascular disease product candidate, C-Cure ${ }^{\circledR}$. The proprietary product consists of autologous bone marrow cells differentiated into cardiopoietic cells with a combination of cytokines and growth factors. CHART-1 trial is a prospective, controlled multicenter, randomized, double-blinded Phase III clinical trial comparing treatment with C-Cure to a sham treatment. The trial recruited 271 evaluable patients with chronic advanced symptomatic heart failure in 12 countries in Europe and Israel. Although there was a positive trend across the complete trial population, no difference between treatment and control (sham procedure) was reached. However, for patients representing $60 \%$ of the overall study population and categorized by their end diastolic volume at inclusion, significance was met for the primary end point, and 
a statistical significant positive difference was seen in all individual elements of the composite primary end point (mortality, worsening heart failure events, quality of life, 6 min walking test, end systolic volume and ejection fraction).

\section{ImmunoCellular}

ImmunoCellular Therapeutics (CA, USA; www.imuc. com) announced that the first patient has been treated in the Phase III trial of ICT-107, a patient-specific, dendritic cell-based immunotherapy targeting multiple tumor-associated antigens on glioblastoma stem cells. The Phase III trial (clinicaltrial.gov identifier: NCT02546102) is a randomized, double-blind, placebo-controlled study of 414 HLA-A2-positive subjects, which will be conducted at approximately 120 sites in USA, Canada and EU. The primary end point in the trial is overall survival, while secondary end points include progression-free survival and safety. ImmunoCellular has also been honored with a US $\$ 19.9$ million award from the governing Board of the California Institute for Regenerative Medicine (www.cirm. ca.gov), California's stem cell agency, to implement the Phase III registration trial. To support timely patient enrollment at participating sites across ten countries, ImmunoCellular has established agreements with the European Organization for Research and Treatment of Cancer (www.eortc.org), the Alliance for Clinical Trials in Oncology (www.allianceforclinicaltrialsinoncology.org) in USA and the Canadian Brain Tumor Consortium (www.cbtc.ca).

Juno

Juno Therapeutics (WA, USA; www.junotherapeutics. com) announced that encouraging clinical data from JCAR015, a CAR T-cell product candidate, support its strategic approach toward the commercialization of its first CAR T therapy. Updated results have been presented at 52nd Annual Meeting of the American Society for Clinical Oncology in Chicago (IL, USA). In a Phase I clinical trial, 46 patients with relapse-remitting B-cell acute lymphoblastic leukaemia received lymphodepleting chemotherapy followed by 19-28z CAR T-cell infusion. Complete response and minimal residual disease-negative complete response rates were 91 and $71 \%$ in the minimal disease cohort, and 75 and 65\% in the morphologic disease cohort, respectively. Additional information about this clinical trial is available in the abstract: http://meetinglibrary.asco.org/content/171505-176 and at http://clinicaltrials.gov (ID: NCT01044069).

\section{Kite Pharma}

Kite Pharma (CA, USA; http://kitepharma.com) delivered three oral presentations relating to its clinical pro- grams at the 2016 European Hematology Association Annual Congress in Copenhagen, Denmark. Updated results from a Phase I clinical trial ZUMA-1 (clinicaltrial.gov identifier: NCT02348216) on the effect of treatment of seven patients with relapsed/refractory aggressive B-cell non-Hodgkin lymphoma with an anti-CD19 CAR T cells (KTEC19) therapy showed no adverse events after 1 month postdosing, with an overall response rate of $71 \%$ and a complete response rate of $57 \%$. The promising results from a Phase I/IIa study conducted as part of a Cooperative Research and Development Agreement between Kite and the National Cancer Institute evaluating anti-CD19 CAR T-cell therapy after low-dose chemotherapy in 22 patients with advanced lymphoma were also presented. Among 19 patients with diffuse large B-cell lymphoma of various subtypes, eight demonstrated complete response, five partial response, two stable disease and four progressive disease. A complete response was also noted in one patient with mantle cell lymphoma. Two patients with follicular lymphoma both obtained complete response. An additional oral presentation featuring data from the SCHOLAR1 study, a meta-analysis of outcomes in patients with chemorefractory diffuse large B-cell lymphoma, showed that response rates after chemotherapy and autologous stem cells transplantation ranged from 19 to $36 \%$ (complete responses from 2 to $18 \%$ ), with a poor median survival, ranging from 4.6 to 6.9 months.

\section{RIKEN}

The Riken Center for Developmental Biology in Kobe (http://www.cdb.riken.jp/en/) will resume a clinical study in which retinal tissues developed from iPSC will be transplanted to a person with an eye disease, in cooperation with Kyoto University and other medical institutes. Additional information on the study can be found at http://www.riken-ibri.jp/AMD/english/ index.html, the International Clinical Trials Registry Platform (http://www.who.int/ictrp/en/) under ID: JPRN-UMIN000011929 and at the Japan Primary Registries Network (http://www.umin.ac.jp/ctr/ index.htm) under ID: UMIN000011929.

\section{San Bio}

SanBio (CA, USA; http://san-bio.com) has published the 12-month interim follow-up data from a 2-year clinical trial of its SB623 stem cell treatment in patients with chronic motor deficits from ischemic stroke [1]. The trial was an open-label, single-arm dose escalation study of 18 patients. Patients had chronic motor deficits more than 6 months following their initial stroke. The group was split into three cohorts that each received different SB623 cell doses. SB623 cells, 
SanBio's proprietary product, are modified allogeneic mesenchymal stem cells, derived from the bone marrow of healthy human adult donors. The data suggest the cell treatment was both well-tolerated and effective in promoting functional recovery in those living with motor impairments 6 months to 5 years following their stroke. Additional information about this clinical trial is available at http://clinicaltrials.gov (ID: NCT02448641).

\section{Stem Cells}

StemCells (CA, USA; www.stemcellsinc.com) has terminated the Phase II 'Pathway' Study in spinal cord injury following an in-depth review of data from the study and after obtaining the concurrence of the study's Interim Analysis Data Monitoring Committee. The Pathway Study was a single blind, randomized, controlled clinical trial investigating the use of StemCells' proprietary human neural stem cells for the treatment of chronic spinal cord injuries. Patients eligible for the study had complete loss of motor control below the level of injury. Although the results showed overall improvement in patients treated with the company's proprietary cells, the magnitude of the effect and the perceived trend of the effect over time did not justify continuing the study or exploring the variability in the initial patient observations, given the financial resources available to the company.

\section{Regulations, approvals, acquisitions...}

\section{Green light}

\section{Cell Cure}

BioTime's (CA, USA; www.biotimeinc.com) subsidiary Cell Cure Neurosciences (Israel; www.cellcureneurosciences.com) received Data Safety Monitoring Board approval to start second patient cohort in a Phase I/IIa clinical trial for advanced dry-form agerelated macular degeneration. Patients with dry-form age-related macular degeneration will receive a higher dose of OpRegen ${ }^{\circledR}$, an allogeneic cell therapy consisting of retinal pigment epithelial cells differentiated from human ESC, to evaluate the safety and efficacy of three different dose regimens of OpRegen (clinicaltrials.gov identifier: NCT02286089). Cell Cure has recently been awarded a new grant for 2016 of 8.4 million shekels (approximately US $\$ 2.2$ million) from the Israel Innovation Authority ('IIA', formerly the Office of the Chief Scientist) of the Ministry of Economy and Industry.

\section{Cytori}

Cytori Therapeutics (CA, USA; www.cytori.com) has recently seen approval of Cytori Cell Therapy ${ }^{\mathrm{TM}}$ for clinical use to treat knee osteoarthritis at the Tokyo Osteoarthritis Clinic under the Japanese 'Act on the Safety of Regenerative Medicine'. Tokyo Osteoarthritis Clinic will begin to treat knee osteoarthritis patients with Cytori Cell Therapy immediately. This approval is limited to facilities under the clinic's umbrella but does not restrict the number of patients that can be treated. Such approvals are not reimbursed by the social healthcare system and patients will be responsible for the costs of treatment.

\section{Stempeutics}

Stempeutics Research (India; www.stempeutics.com) announced that the Drugs Controller General (India) has granted limited approval for manufacturing and marketing of proprietary product Stempeucel ${ }^{\circledR}$ for the treatment of critical limb ischemia due to Buerger's disease. Stempeucel consists of ex vivo cultured adult allogeneic mesenchymal stromal cells isolated from multiple bone marrow donors, thus would become the first allogeneic product approved in India.

\section{Guidance}

Japan

The Japanese health ministry has drawn up guidelines for determining the safety of laboratory-created stem cell treatments in clinical trials, a step that could pave the way for more such studies. The standards will be used to evaluate trials that involve transplanting iPSC into the human body. The safety standards call for testing these iPSC for signs that they may turn cancerous. Ideally, only cells without cancer-causing abnormalities should be used, but exceptions may be made in cases where the potential benefits of the trial outweigh the risks, according to the guidelines. The cells first would be transplanted into nonhuman subjects, such as rats, to check for cancer and other potential complications.

\section{NCMC}

The Georgia Research Alliance (GA, USA: http://gra. org) and the Georgia Institute of Technology (GA, USA; www.gatech.edu) have launched the National Cell Manufacturing Consortium (http://cellmanufacturingusa.org), an industry-academic-government partnership that recently released the National Roadmap for Advanced Cell Manufacturing (http://cellmanufacturingusa.org/road-map). Establishment of the consortium and development of this 10-year national roadmap was sponsored by the National Institute of Standards and Technology (NIST; MD, USA; www.nist.gov). 
Established in 2014 through an NIST Advanced Manufacturing Technology grant, the National Cell Manufacturing Consortium is an industry-driven consortium including cell manufacturing experts from industry, academic research, clinical GMP centers, government agencies and private foundations.

\section{Capital market \& finances CIRM}

The governing Board of the California Institute for Regenerative Medicine (CIRM; CA, USA; (www. cirm.ca.gov) approved investing US $\$ 15$ million in a partnership with Quintiles Transnational (CO, USA; www.quintiles.com) to create a stem cell Accelerating Center. In return, Quintiles will provide researchers with the support and management services they need to increase the likelihood their clinical trials will succeed. CIRM projects run through the Accelerating Center will be given a discount on services. CIRM expects to receive a US $\$ 22.5$ million, or $50 \%$, return on its US\$15 million investment.

\section{Mesoblast}

Mesoblast (Australia; www.mesoblast.com) has regained full worldwide rights and full strategic flexibility to exploit its mesenchymal precursor cell technology platform for the cardiovascular field as Teva Pharmaceutical (Israel; www.tevapharm.com), Mesoblast's key US partner, surrendered all rights to its heart failure treatment, leaving the local company to fund as much as US $\$ 100$ million in medical trials before it could be ready for marketing. In closing trading, Mesoblast shares were down $42 \%$ at US $\$ 1.11$, wiping more than US\$300 million off its market value and taking the shares to 7 year lows. Teva's decision follows the failure of Celgene to exercise a 6-month option to partner some of Mesoblast's research programs, which was only disclosed recently. The lead asset in this cardiovascular portfolio is Mesoblast's Phase III product candidate MPC-150-IM for advanced chronic heart failure. The Phase III trial has enrolled over 230 patients out of a total of 600 patients with advanced heart failure, with the primary end point agreed to with the
US FDA for product registration being recurrent heart failure-related hospitalizations or deaths.

\section{Competitions}

NASA

NASA (USA; www.nasa.gov), in partnership with the nonprofit Methuselah Foundation's (VA, USA; www. mfoundation.org) New Organ Alliance (www.neworgan.org), is seeking ways to advance the field of regenerative medicine through a new prize competition. The Vascular Tissue Challenge offers a US $\$ 500,000$ prize to be divided among the first three teams that successfully create thick, metabolically functional human vascularized organ tissue in a controlled laboratory environment. Related cells that are joined together are collectively referred to as tissue, and these cells work together as organs to accomplish specific functions in the human body. Blood vessels around the cells vascularize, providing nutrients to the tissue to keep it healthy. The vascularized, thick-tissue models resulting from this challenge will function as organ analogs, or models, that can be used to study deep space environmental effects, such as radiation, and to develop strategies to minimize the damage to healthy cells. Studying these effects will help create ways to mitigate negative effects of space travel on humans during long duration, deep space missions.

\section{Financial \& competing interests disclosure}

The authors have no relevant affiliations or financial involvement with any organization or entity with a financial interest in or financial conflict with the subject matter or materials discussed in the manuscript. This includes employment, consultancies, honoraria, stock ownership or options, expert testimony, grants or patents received or pending, or royalties.

No writing assistance was utilized in the production of this manuscript.

\section{Reference}

1 Steinberg GK, Kondziolka D, Wechsler LR et al. Clinical outcomes of transplanted modified bone marrow-derived mesenchymal stem cells in stroke: a Phase 1/2a study. Stroke 47(7), 1817-1824 (2016). 